\title{
The grand challenges in electrochemical corrosion research
}

\author{
Guang-Ling Song * \\ Materials Science and Technology, Oak Ridge National Laboratory, Oak Ridge, TN, USA \\ *Correspondence: guangling.song@gmail.com \\ Edited and reviewed by: \\ Wei Gao, The University of Auckland, New Zealand
}

Corrosion is the process of degradation or failure of a material resulting from a chemical reaction between the material and its surrounding environment. It is an indispensible research area in material science and engineering, because corrosion resistance characterizes the stability or durability of a material, which is one of the most important material performances in application.

Compared with other materials, metals are relatively active, and likely to be susceptible to corrosion attack. Corrosion research thus mainly deals with the damaging mechanism and behavior of various metals, including ferrous or nonferrous, single crystal or nano-crystalline, cast or wrought, and structural or functional alloys. It has naturally grown into different branches based on alloy (Shreir et al., 1994), such as steel corrosion, Al alloy corrosion, Ni alloy corrosion, etc. The composition and microstructure are always the most decisive factors in determining the corrosion resistance of an alloy. On this aspect, a critical issue to address is how corrosion process is affected by matrix phase composition, segregated alloying element, lattice structure, crystalline defect, crystal orientation, grain size, secondary phase constituent, inter-metallic particle distribution, porosity, micro-crack density, impurity level, and surface state.

Another important aspect of corrosion research is the complicated influence of environmental factors on corrosion. The sensitivity of corrosion behavior to environmental factors has led to varied degrees and forms of damage of metals under different service conditions (Cramer and Covino, 2006). According to the medium that metals are exposed to, corrosion can be easily classified into different types, such as aqueous or non-aqueous corrosion, ambient or high temperature corrosion, acidic or alkaline corrosion, etc. In the natural environment, atmospheric corrosion, seawater corrosion, and underground corrosion usually attack metals differently. A variety of corrosion problems associated with service environments have also been frequently reported in chemical, oil/gas, pipeline, civil, auto, aerospace, military, nuclear, and medical industries. As most environmental factors, like temperature, pressure, chemical composition, constituent concentration, $\mathrm{pH}$ value, electrical or thermal conductivity, viscosity, etc., can directly or indirectly interact, and continuously or inconstantly influence a corrosion process, the prediction of longterm corrosion behavior is quite difficult. Identifying a key influencing factor and understanding its effect on corrosion kinetics would be a research focus on this aspect.

The core of corrosion research focuses on the material-environment reaction mechanism. It is the fundamental understanding of the detailed reaction processes, procedures, and steps, as well as their influencing factors that underpins corrosion science. Most ambient corrosion problems can be ascribed to electrochemical reactions (Kaesche, 2003), because moisture and aqueous liquid are widely present in the natural environment, and an electrochemical reaction is generally faster than oxidation-reduction reactions under ambient conditions. Stress-induced corrosion failure under many circumstances is a result of complicated interactions between stress and electrochemical reactions; the stress dramatically facilitates the electrochemical process and the latter strikingly amplifies the former's damaging effect. Even at high temperatures, the molten salt corrosion may also be described as an electrochemical process. Therefore, electrochemistry is one of the most pertinent subjects in corrosion research (Mansfeld and Bertocci, 2005). To gain an insight into the core area of corrosion science, investigation of detailed electrochemical mechanisms and establishment of metal-electrolyte interface models should be prioritized.

Having gone through the basic aspects and the core of corrosion science, one should never forget that the ultimate goal of corrosion research is to minimize corrosion damage. In this regard, all the methods that can interfere with the metal-environment reaction and effectively slow down the corrosion process are of great interest. In fact, cathodic protection (Baeckmann et al., 1997), coating (Swaraj, 1996), surface treatment/modification (Biestek and Weber, 1976), and inhibitor (Braford, 1993) techniques that retard the corrosion through different mechanisms have a long history of successfully mitigating corrosion damage in practice. They have built up an important extension of corrosion science, which more or less overlaps surface and coating science, technology, and engineering. Although technological innovation is the central theme in this area, scientific breakthroughs are highly desired. Innovations in corrosion prevention often emerge when new ideas, techniques, and results from other disciplines are introduced into this applied field of corrosion research.

From the above brief introduction, the basic characteristics that virtually differentiate corrosion research from the other disciplines in materials science and engineering can be outlined as follows:

(1) Corrosion occurs in a complicated system simultaneously influenced by environmental factors and metallurgical parameters. These interactive factors tremendously complicate the corrosion system. Thus, the key influencing factor and the rate-determining step that govern the fundamental kinetics of a corrosion process cannot be clearly identified. Consequently, many corrosion phenomena cannot be interpreted theoretically. Corrosion 
research in some cases has to rely on a substantial body of empirical knowledge.

(2) Corrosion is a time-sensitive process. Since most environmental factors can vary markedly with time, the corrosion performance of a metal may be unbelievably dissimilar in different stages. Even in a corrosion system with constant environmental factors and strictly controlled initial and boundary conditions, the corroding surface state can change with time due to dissolution of metal or deposition of corrosion products. Hence, the varying environmental parameters compounded with the complicated influence of environmental factors make theoretical prediction of corrosion extremely difficult.

(3) There is a lack of high-resolution in situ techniques to reveal the corrosion mechanism. Due to the dependence of corrosion on environmental conditions, ex situ measurements may not generate essential information of a corrosion process or may distort the information. Currently, most of the advanced instruments popularly used in characterizing the microstructure and composition of a metal surface at a nano level must operate in high vacuum, or require the sample to be carefully pre-treated/prepared, or need addition of some supporting species to provoke additional reactions in the corrosion system. They cannot produce real in situ results from an undisturbed corroding surface in aggressive media. It is expected that some new emerging high-resolution in situ methods, e.g., a specially designed electrolyte cell that can be installed in SEM or TEM, will become available for most corrosion researchers in investigating the microstructure and composition of a corroding surface in liquid media.

On top of these basic characteristics of corrosion research and resultant difficulties, there are many challenging issues to address, some of which have become substantial hurdles critically impeding further development of corrosion science. Successfully solving these critical problems may significantly advance this field. For example, the following topics are particularly challenging, which may interest researchers at the forefront of corrosion science.

(1) Corrosion of metastable metals: while material science is spreading into nano-technology and metastable microstructures like amorphous and nano-crystalline alloys, current corrosion principles stemming from traditional crystalline metals start to face new challenges. Understanding the corrosion reaction on a surface consisting of an extremely high density of defects is a new area in corrosion science. To extend and deepen corrosion knowledge and enable practical applications of the innovative materials, the challenge of developing new corrosion theories for a highly activated metal surface must be met first.

(2) Corrosion and protection of advanced engineering metals: as the demand increases for materials with some highly desired properties, a few alloys that were originally deemed to be unsuitable for some applications are now becoming shortlisted candidates. For example, $\mathrm{Mg}$ alloys were initially too active to be popular structural materials, but now the high strengthweight ratio makes them attractive in the auto industry as candidate materials for the next generation of vehicles. These kinds of materials usually have a corrosion behavior different from traditional alloys, and existing corrosion and protection knowledge and technology cannot be directly applied to them. It is important and challenging to further develop the existing corrosion principles and techniques to cover these advanced engineering alloys.

(3) Detailed mechanism of the effect of nanostructure on corrosion: the corrosion resistance of an alloy is fundamentally determined by its surface "weak points." These active sites on the surface are actually crystalline defects, such as dislocations, twins, grain boundaries, phase interfaces, segregated alloying elements, precipitated intermetallics, or impurity inclusions. Understanding the corrosion reaction details in these sites is obviously an inevitable step in solving the central problem of corrosion science. Unfortunately, these defects are usually at nano scale. It is a big challenge to reveal the micro corrosion mechanism in these defective sites before high-resolution in situ experimental techniques are mastered by corrosion scientists.

(4) Corrosion mechanism and prevention technology under extreme conditions: the extreme conditions refer to extraordinarily strong interferences of electric and magnetic fields, irradiation, pressure, and temperature, etc. Corrosion damage under these severe conditions has been found in nuclear, aerospace, gas/oil, and chemical industries. However, before researchers are equipped with high-resolution in situ techniques particularly designed for these extreme environments, their understanding of this kind of corrosion cannot be as comprehensive as that of ambient corrosion. Also, owing to the harshness of the service environments and the limitation of industrial operational practices, these industries are desperately short of cost-effective corrosion prevention measures. Since this kind of corrosion may ultimately threaten public safety, it is a corrosion scientist's obligation to accept the challenge to improve the understanding of the corrosion under extremes and develop better prevention techniques.

(5) In vivo corrosion understanding, monitoring, and simulation: the human body is a complicated, self-maintained corrosive system. In vivo corrosion is a biodegradation process occurring in the very well-controlled ambient environment. Real-time in situ corrosion measurements or monitoring without disturbing the physiological reactions inside the human body is difficult. The intricate influence of physiological reactions and body fluid constituents on corrosion is another obstacle to understanding the in vivo corrosion mechanism. It is also unlikely to precisely simulate a real in vivo environment through an imitation of the body fluid and physiological reactions in the lab. Therefore, the investigation of biodegradation mechanism and in vivo corrosion process in the lab will remain a challenge in corrosion research. 
(6) Corrosion prediction in the real world: in a natural environment, spontaneous corrosion conditions are not controlled. Moreover, there are myriads of changes of environmental factors that can affect corrosion, which to a great extent complicates the corrosion process. Even worse, the inevitable life activities in the real world can also interfere with the corrosion process, which makes the corrosion behavior nearly unpredictable. For example, the micro-bio/bacteria colonizing in sewage systems, the biofilm building up on offshore infrastructures, the air pollution by auto exhausts, and the contamination of soil/underground water by everyday wastes can all unexpectedly speed up the corrosion of exposed facilities. An unforeseen corrosion of an infrastructure underground, in concrete or in water could lead to a catastrophe under certain circumstances. To reduce the risk, predicting corrosion performance in natural environments is deemed to be a big challenge in corrosion research. On this aspect, establishment of a longterm corrosion database is an essential task, and computational modeling can be an approach that may lead to a breakthrough in corrosion prediction.

(7) Surface state transition mechanism: onset of passivation, initiation of pitting, and breakdown of surface film are examples of surface state transition. There are many more these kinds of surface changes in practice. The transition from the original surface to a new steady state is a transient process. The new state normally depends on the initial surface conditions, which can eventually influence the overall corrosion damage. For example, the rupture of an initially formed film can to a great extent influence the film growth behavior in a later stage; the pitting distribution on a metal surface in an early stage will affect the final corrosion morphology; the initial surface cleanness of steel may sometimes determine the steel passivity. It is of great interest to learn how, when, and where the transition suddenly starts. Predicting the occurrence of the transition on a metal surface is difficult, but it is a challenging goal that must be attained in corrosion research.

(8) Development of corrosion-resistant functional coatings: coatings can completely alter the properties of a metal surface, which opens up many new application fields for metals. Improved corrosion resistance or protectiveness is traditionally an essential requirement for coating development in corrosion prevention engineering. Generally speaking, the corrosion resistance of a coating is a function of the stability of the coating material, the integrity and thickness of the coating layer, and the adhesion of the coating on metals. It also depends on how the coating responds to temperature, stress, and abrasion in service. A corrosionresistant coating is usually a compromise of many requirements. If a new coating can be made to be electrical or thermal insulating or conducting, antiskidding or self-lubricant-releasing, super-hydrophobic or hydrophilic, selectively adsorptive and absorptive to certain species, etc., it will find a much wider application itself and also significantly extend the applications of metallic components/parts in industry. However, such a coating will have too many contradicting requirements to compromise. Its invention and development is not only challenging, but also exciting.

In summary, corrosion as a material degradation process caused by the reaction of the material with its surrounding medium has been intensively and extensively investigated. Many achievements have been made regarding alloy composition and microstructure effect, environmental factor influence, corrosion mechanism, and protection techniques. However, the detailed in situ corrosion mechanism influenced by numerous varying factors has not been comprehensively revealed due to the lack of high-resolution in situ methods. At present, the biggest difficulty in corrosion research is to gain a deep understanding of the degradation process in situ on a corroding surface that is concealed by corrosive media. Around this, there are at least eight grand challenges on the frontiers of corrosion research. Challenging these hot topics may lead to breakthroughs in the forefront of corrosion science.

\section{REFERENCES}

Baeckmann, W., Schwenck, W., and Prinz, W. (1997). Handbook of Cathodic Corrosion Protection, 3rd Edn. Houston, TX: Gulf Professional Publishing.

Biestek, T., and Weber, J. (1976). Electrolytic and Chemical Conversion Coatings. Redhill: Portcullis Press Limited.

Braford, S. A. (1993). Corrosion Control. London: Van Reinhold.

Cramer, S. D., and Covino, B. S. Jr. (eds) (2006). ASM Handbook-Volume 13C: Corrosion: Environments and Industries. Materials Park, OH: ASTM International.

Kaesche, H. (2003). Corrosion of Metals, Physicochemical Principles and Current Problems. Berlin: Springer.

Mansfeld, F., and Bertocci, U. (eds) (2005). Corrosion Tests and Standards, 2nd Edn. West Conshohocken, PA: ASTM International.

Shreir, L. L., Jarman, A. A., and Burstein, G. T. (eds) (1994). Corrosion-Volume 1: Metal/Environment Reactions. Boston, MA: Butterworth Heinemann.

Swaraj, P. (1996). Surface Coatings: Science and Technology, 2nd Edn. Chichester: John Wiley \& Sons.

Received: 13 February 2014; accepted: 25 February 2014; published online: 10 March 2014.

Citation: Song G-L (2014) The grand challenges in electrochemical corrosion research. Front. Mater. 1:2. doi:10.3389/fmats.2014.00002

This article was submitted to Corrosion Research, a section of the journal Frontiers in Materials.

Copyright (c) 2014 Song. This is an open-access article distributed under the terms of the Creative Commons Attribution License (CC BY). The use, distribution or reproduction in other forums is permitted, provided the original author(s) or licensor are credited and that the original publication in this journal is cited, in accordance with accepted academic practice. No use, distribution or reproduction is permitted which does not comply with these terms. 